\title{
Comparative ergonomic workflow and user experience analysis of MRI versus fluoroscopy-guided vascular interventions: an iliac angioplasty exemplar case study
}

Fabiola Fernández-Gutiérrez, Santiago Martínez, Martin A. Rube, Benjamin F. Cox, Mahsa Fatahi, Kenneth C. Scott-Brown, J.

Graeme Houston, Helen McLeod, Richard D. White, Karen French, Mariana Gueorguieva, Erwin Immel, Andreas Melzer

This is the authors' final version of this article. The final publication is available at Springer via http://dx.doi.org/10.1007/s11548-015-1152-y 


\section{Title Page}

Manuscript title: Comparative ergonomic workflow and user experience analysis of MRI versus fluoroscopy guided vascular interventions: an iliac angioplasty exemplar case study.

\section{Authors:}

Fabiola Fernández-Gutiérrez, $\mathrm{PhD}(1)$

Santiago Martínez, $\mathrm{PhD}(2,3)$

Martin A. Rube, $\mathrm{PhD}(1)$

Benjamin F. Cox, MD (1)

Mahsa Fatahi, MSc (1)

Kenneth C. Scott-Brown, PhD (2)

J. Graeme Houston, MD, FRCP, FRCR, EBIR $(4,5)$

Helen McLeod, BSN (5)

Richard D. White, MBChB, FRCR $(4,6)$

Karen French, BSN (1)

Mariana Gueorguieva, $\mathrm{PhD}(1)$

Erwin Immel, MSc (1)

Andreas Melzer, MD, DDS (1)

\section{Affiliations:}

(1) Institute for Medical Science and Technology, Division of Imaging and Technology, University of Dundee, Dundee, United Kingdom

(2) Centre for Psychology, Abertay University, Dundee, United Kingdom

(3) eHealth and Healthcare Centre, Faculty of Health and Sport Sciences, University of Agder, Grimstad, Norway

(4) Department of Clinical Radiology, Ninewells Hospital and Medical School, NHS Tayside, Dundee, United Kingdom

(5) Medical Research Institute, The Institute of Cardiovascular Research, University of Dundee, Ninewells Hospital and Medical School, Dundee, United Kingdom

(6) Department of Clinical Radiology, University Hospital of Wales, Cardiff, UK

\section{Corresponding author:}

Fabiola Fernández-Gutiérrez

Institute for Medical Science and Technology

Wilson House, 1 Wurzburg Loan

DD2 21FD, UK

Phone: +44 (0)77380 72016, Fax: +44 (0)1382 386588

fabiola.fg@gmail.com 


\section{ABSTRACT:}

Purpose: A methodological framework is introduced to assess and compare a conventional fluoroscopy protocol for peripheral angioplasty with a new magnetic resonant imaging (MRI)-guided protocol. Different scenarios were considered during interventions on a perfused arterial phantom with regards to time-based and cognitive task analysis, user experience and ergonomics.

Methods: Three clinicians with different expertise performed a total of 43 simulated common iliac angioplasties (9 fluoroscopic, 34 MRI-guided) in two blocks of sessions. Six different configurations for MRI guidance were tested in the first block. Four of them were evaluated in the second block and compared to the fluoroscopy protocol. Relevant stages' durations were collected and interventions were audio-visually recorded from different perspectives. A cued retrospective protocol analysis (CRPA) was undertaken, including personal interviews. In addition, ergonomic constraints in the MRI suite were evaluated.

Results: Significant differences were found when comparing the performance between MRI configurations versus fluoroscopy. Two configurations (with times of 8.56(0.64) and 9.48(1.13) minutes) led to reduce procedure time for MRI guidance, comparable to fluoroscopy (8.49(0.75) minutes). The CRPA pointed out the main influential factors for clinical procedure performance. The ergonomic analysis quantified musculoskeletal risks for interventional radiologists when utilising MRI. Several alternatives were suggested to prevent potential low-back injuries.

Conclusions: This work presents a step towards the implementation of efficient operational protocols for MRI-guided procedures based on an integral and multidisciplinary framework, applicable to the assessment of current vascular protocols. The use of first-user perspective raises the possibility of establishing new forms of clinical training and education. 
Keywords: Interventional MRI workflow, MR-guided angioplasty, retrospective protocol analysis, ergonomic analysis

\section{INTRODUCTION}

Magnetic resonant imaging (MRI) guided vascular interventions could be a favourable alternative to the conventional fluoroscopic guidance due to added diagnosis value of having a high soft tissue contrast without exposing patients and clinicians to ionising radiation [1]. However, MRI environments present operational challenges that need to be addressed in order to make MRI guided procedures comparable to fluoroscopy in terms of safety, efficiency and efficacy, and acceptable for clinical practice. Much of the current published research focuses on overcoming technical limitations and safety issues [2, 3]. In addition, concerns on the potential longer procedural times have been reported in previous studies [4]. Safety and efficiency can be improved by objective analysis of a procedure subdivided in tasks along with a detailed assessment of other components of the workspace (e.g. communication, cognitive load) [5]. In addition, these observational studies provide a better understanding of surgical teams by examining the underlying principles that can contribute to medical errors [6]. Two studies have been conducted in the field of interventional radiology. Johnson et al. [7] presented a cognitive task analysis on several fluoroscopy-guided procedures in order to incorporate the acquired knowledge to better simulate models for training. Van Herzeele et al. [8] applied this concept to a simulator for fluoroscopic treatment of iliac stenoses, comparing trainees and experts.

In our project, we applied the fundamentals of time-based and cognitive task analysis (i.e. time task analysis deals with how a task is accomplished, essentially through collection of task durations; cognitive task analysis uses a variety of interviews and observations to extract knowledge when users perform complex tasks) to develop an efficient operational protocol in an MRI environment. However, the MRI context is substantially different from angiography 
suites in terms of patient access, equipment, physical space available for clinicians, and significant image acquisition and visualisation differences. For these reasons, this study incorporated a cued retrospective protocol analysis (CRPA) as part of the cognitive analysis [9]. Through the CRPA, we audio-visually recorded the first-person perspective of the clinician's activity to retrospectively observe the steps followed and investigate the information necessary to accomplish the task.

MRI environments for endovascular procedures create additional challenges such as the potential occupational hazards that clinicians may face during interventions, e.g. risk of a musculoskeletal injury [10]. Fatigue and uncomfortable postures during the work activity can reduce concentration, increasing the possibility of medical errors and risk for the patient [11]. Recent studies highlight the importance of ergonomic analysis when designing new imaging environments for vascular procedures $[12,13]$. Restricted access to patients and limited space in scanner rooms are some of the limiting factors to be considered when analysing ergonomics in MRI suites.

This current study introduces the use of MRI guidance for vascular procedures by comparing a fluoroscopy driven standard workflow protocol with a proposed MRI-guided protocol using as exemplar a case of peripheral angioplasty. The technology, devices and MRI protocols involved in this work and a detailed description of the environmental setup were first presented in Rube et al. [14]. In contrast, this paper focuses in the procedural protocol evaluation, which is done from multiple viewpoints with regards to previously cited authors $[2,3,8]$. The framework included a time-based and cognitive task analysis via CRPA to assess clinical performance in several different scenarios using the MRI-guided protocol. Finally, we present a simulation-based ergonomic analysis on key postures held by clinicians during interventions, offering additional insights by simulating a wide range of alternatives to overcome ergonomic issues [15]. 


\section{METHODS AND MATERIALS}

\subsection{Procedure}

A total of 43 simulated uncomplicated percutaneous transluminal angioplasties of the iliac artery (PTA-IA) were performed in an arterial vessel phantom (9 under fluoroscopy and 34 under MRI guidance). The aims were: 1) to identify and evaluate procedural differences between a fluoroscopy-guided and an MRI-guided procedure; and 2) to analyse the potential effects on the performance and clinicians' experience, understood as their perception of the interventional environment, during vascular interventions.

As a baseline, we adapted the standard protocol for PTA-IA followed in our local clinical radiology department, shown in Figure 1a. Figure $1 \mathrm{~b}$ presents the alternative protocol proposed for the MRI-guided procedures, intentionally designed to be similar to fluoroscopy driven procedure aligned with current standards. Total arterial occlusion or tasks related to the phantom preparation were not taken into account in the study, as they were not relevant for the interventional tasks.

\subsection{Facilities and equipment}

Interventions were performed at local imaging research facilities, including an angiography suite, equipped with a digital subtraction angiography (DSA) unit (OEC 9900 Elite, GE Medical Systems, Waukesha, WI, USA) and an adjacent MRI scanner room, with a $1.5 \mathrm{~T}$ MRI scanner (Signa HDxt, GE Medical Systems, Waukesha, WI, USA)..

In addition to the standard control console for the MRI scanner, another workstation was installed with a real-time MRI software framework (RTHawk, Version 0.9.28, HeartVista, Inc., Los Altos, CA, USA) [16]. A 40" LCD monitor and an MRI-safe mobile touchscreen device (iPad 1, Apple Inc., Cupertino, CA, US) were installed in the MRI suite. To enable physician/operator (scanner/control room) communication during the procedures, we used a second tablet device and Bluetooth earphones positioned under the noise protection earmuffs. 
IP webcams were placed in different positions with respect to the MRI scanner: right, left, and in-bore (Figure 3a-c). Radiologists wore recording spectacles for a first-person experience evaluation (Figure 3d). The arterial vessel phantom (Elastrat, Sarl, Switzerland) was connected to a heart-lung machine to mimic (pulsatile) physiologic flow. We customised commercially available non-braided balloon catheters by attaching a resonant circuit in order to visualise them under MRI.

Rube et al. [14] describes in more detail the experimental setup, also included in the Appendix.

\subsection{Methodology}

Two clinicians with different expertise level carried out the experiments: a senior interventional radiologist (consultant) with more than 20 years of experience in vascular procedures and MRI diagnostic (A) and a trainee physician with no experience in clinical interventional radiology (B). Both clinicians were familiar with MRI environments and the facilities prior to this study. A nurse with experience in interventional radiology techniques assisted the clinicians during all experiments.

Experiments were carried out in two separate blocks of sessions. In the first block, each clinician (A and B) performed three repetitions (6 in total) of PTA-IA under fluoroscopy guidance following the adapted protocol presented in Figure 1a. Afterwards, during the first block, we performed a pilot study in the MRI environment and clinicians were asked for qualitative feedback on six different configurations that were tested for the procedures under MRI guidance (see Table 1). A third clinician, final-year specialty trainee radiologist with 3 years' experience in vascular procedures but no experience in MRI, participated during the first session to provide additional appraisal in the different setups but did not participate in subsequent sessions. The changes considered in the setups consisted of: varying the workstation controlling the scanner (RTHawk or Standard Interface (GE iDrive)); varying the 
in-room visualisation equipment; and whether or not use Bluetooth earphones for communication between the scanner and control rooms.

After the first block, clinicians reported the need for communication between scanner and control room (clinician/controller) during the intervention. As a result, the second block of experiments evaluated only four configurations - I to IV in Table 1-incorporating in all the preferred two-way voice communication (i.e., Bluetooth earphones).

During the first block, times were collected for the stages of the fluoroscopy-guided procedures. Similarly, times were collected for the MRI-guided procedure stages during the second block. Additional information was compiled such as discussions between the teamwork and any difficulty found with the vascular model or devices. All procedures were audio-visually recorded from third and first person perspectives, the latter using high definition recording spectacles.

\subsection{Data analysis}

The generalised estimating equations (GEE) method for repeated measures was used to analyse the complete dataset after the second block of experiments [17]. Since, tasks completion times are usually non-normal distributed data [18], gamma distribution was assumed. In addition, first-order autoregressive correlation was considered as a robust design measure for the GEE analysis.

Data analysis revealed that values of several variables were mistaken or unavailable $(13.5 \%$ of a total of 318 values collected). Due to the low number of repetitions for each configuration ( $\mathrm{n}=2$ or 3 depending on the case) and since data were missing at random (MCAR), multiple imputation (MI) was used to generate the missing values . Five imputed datasets were created using the fully conditional specification approach in IBM SPSS v21.0.0 (New York, USA) [19].

\subsection{Cued retrospective protocol analysis}


A CRPA including interviews and commentary analysis was carried out with clinicians A and B, as participants of both blocks of sessions (Figure 4a). We used recorded oral descriptions by clinicians when simultaneously visualising their own audio-visual recordings of the operations in first-person (i.e., HD spectacles camera) and third-person perspectives (i.e., front, rear and bore). In total, 4 perspectives were concurrently shown (see Figure 3) in one large screen (3200x1200 resolution with a length of 5.7 metres). Clinicians visualised their own operation and freely orally commented what was being seen and any other information considered relevant to understand the scenes (Figure $4 \mathrm{~b}$ ).

As an additional information gathering exercise, clinicians wore a head mounted iView-X HED eye movement recording device (SensoMotoric Instruments GmbH (SMI), Warthestraße, 21D-14513, Teltow, Germany). This system allows for free head movement during commentary and records the eye gaze position at $30 \mathrm{~Hz}$ frequency with an accuracy of 0.5 degrees of visual angle. A 5-point calibration protocol was conducted to ensure accurate recordings by participants looking at each corner of the monitor and the centre while the experimenter registers eye position on the associated iView software. The resulting recordings provide a first person perspective video with overlaid gaze cursor, used by the experimenter in the review of the CRPA to inform the viewer of the gaze associated with individual elements of the task. CRPA recordings were reviewed by two of the authors independently (Fernandez-Gutierrez and Martinez) to identify the factors that influenced the performance of all procedures.

\subsection{Ergonomics analysis}

CRPA interviews and multi-video recordings were used to identify clinicians' perceptions about postures in the MRI environment. A RULA (Rapid Upper Limb Assessment) analysis was implemented over the positions identified [20]. 
RULA gives scores from 1 (the posture is acceptable) to 7 (changes are required immediately). This global score is calculated by grouping to sets of individual scores: a first group with neck, trunk and legs; and a second group with upper arm, lower arm, wrist and wrist twist. Effects of muscles and forces required to maintain the posture are also considered. To interpret these scores, the RULA analysis provide a colour coding: green for scores of 1-2, yellow for 3-4, orange for 5-6 and red for 7 .

To compare the effect of different scenarios in the postures, we implemented a simulated 3D environment in Delmia V5R20 for Human Ergonomics (Dassault Systèmes S.A., VélizyVillacoublay, France), using a $50^{\text {th }}$ percentile male mannequin $(173 . \mathrm{cm}$ height and $76.20 \mathrm{Kg}$ weight [21]) as DHM. The usual posture adopted by clinicians in angiography suites, defined as initial position (position 1), was set as baseline to compare with the MRI scenarios. The analysis classified the type of activity as static (position held for more than one minute); intermittent (position held less than one minute) or repeated (position repeated a minimum of three times during the activity).

The RULA analysis was applied to the postures on two CAD (Computer-aided design) models: a 1.5T Signa MRI scanner model (GE Healthcare, Waukesha, WI, USA) with a $60 \mathrm{~cm}$ diameter bore, as scanner used during the experiments; and a $70 \mathrm{~cm}$ wide bore $3 \mathrm{~T}$ Discovery MRI scanner model (GE Healthcare, Waukesha, WI, USA). In addition, three more scenarios were compared to assess potential improvement in the comfort of the postures by adding an arm-supporting device and an adjustable platform for personalised height.

\section{RESULTS}

\subsection{Tasks analysis}

In total, 43 procedures were recorded, 19 during the first block of sessions and 24 during the second block. Table 2 presents the total procedure times in minutes collected for the fluoroscopic interventions during the first block and per MRI configuration per clinician (A 
and B) during the second block. The mean total duration was 12.08(0.95) (mean(standard error - SE) minutes (min) per procedure.

The overall performance of clinician A was significantly $(\mathrm{p}<0.001)$ faster than clinician $B$, taking the first one an average of 11.43 (1.43) min versus the 12.74 (1.27) min of clinician B. When comparing the different configurations of MRI guidance versus the standard fluoroscopy protocol, the GEE analysis revealed significant difference $(\mathrm{p}<0.05)$ when the GE iDrive was used (GEScreenBT and GEiPadBT in Table 1), and also when the RTHawk and iPad were used together (RTiPadBT in Table 1$)$. There was no significant difference $(p>0.05)$ in the overall performance of the standard fluoroscopy protocol $(8.49(0.75) \mathrm{min})$ when compared with RTHawk using the LCD in-room monitor (8.56(0.64) min).

Additionally, a more detailed analysis of the stages indicated in Figure 1 was performed, with the ones considered more relevant for the study reported here. The treatment phase was defined from the moment the balloon catheter was inserted until the moment the balloon was extracted after inflation. Configurations II - GEiPadBT and IV - RTiPadBT were significantly different $(\mathrm{p}<0.05)$ when compared with the performance under fluoroscopic guidance. As seen in Table 3, in GEiPadBT times were on average faster (3.14(0.28) min) than in fluoroscopy $(3.63(0.27) \mathrm{min})$, while RTiPadBT took longer $(4.25(0.45) \mathrm{min})$. However, although GEiPadBT was slightly faster than fluoroscopy for the treatment times, overall GEScreenBT and GEiPadBT were slower than the others (see Table 2). This is explained when looking at the pre- and post-angiography times (see Table 3). In GEScreenBT and GEiPadBT, these phases took significantly $(\mathrm{p}<0.01)$ longer than in the fluoroscopically-guided procedure. On the contrary, in RTScreenBT and RTiPadBT, these times were similar.

\subsection{Cognitive and user experience analysis}


Figure 5 provides an overview of the factors most frequently discussed by clinicians A and B during the interviews. The importance level of these factors was qualitatively classified by the number of times they were referred to during the interviews and the emphasis given by the clinicians, graphically indicated by the size of the particular bubble with their name in the figure. In addition and for clarity, these factors were primarily grouped according to their nature: communication, visualisation and ergonomics. The diagram also shows the hierarchical dependency within the groups (black arrows) and the interrelations among different groups (red arrows). In a general evaluation, communication appeared as the most important factor during all procedures, followed by visualisation. Ergonomics inside the room were important for the clinicians to a lower extent. Specifically within the groups, communication with the control room was ranked more relevant than the communication inside the room. Clinicians designated the visualisation of devices as critical during the procedures. Moreover, the type of screen played an important role. With less level of importance, clinicians appreciated that the acquisition of MRI images should be improved as differences were encountered when compared to DSA. By contrast, clinicians agreed on the importance of temporal and spatial resolution of interventional MRI images, rated as sufficient with the current MRI pulse sequences used in our proposed protocol.

\subsection{Evaluation of ergonomics}

The information gathered by the multiple video recordings showed that clinicians maintained ergonomically disadvantageous postures while carrying out the procedures under MRI in comparison with the performance in the angiography suite. As a result, we carried out an ergonomic analysis of the MRI environment, whose fundamentals were based on a preliminary study [22].

Four key positions were identified as being repeatedly adopted by the clinicians during the MRI-guided procedures: one resting position (position 1) and three operating positions 
(positions 2-4). Figure 6 presents the four postures and the corresponding DHMs for the two scenarios analysed, using the $1.5 \mathrm{~T}$ GE Signa scanner with $60 \mathrm{~cm}$ bore $(6 \mathrm{~b})$ and a $3 \mathrm{~T}$ GE Discovery scanner with $70 \mathrm{~cm}$ bore (6c). RULA global scores associated to each position are given below. Position 1 was considered static, position 2 intermittent and the rest repeated. The scores showed very small differences between the postures held using both scanners and indicate that positions 2-4 are ergonomically not acceptable for day-to-day practice.

Table 4 shows results of the comparison between the RULA scores obtained in the initial test with three alternative scenarios considered: adding an arm-support, an adjustable height platform and a combination of both. With an adjustable height platform, mannequin's height was reduced until a comfortable position for the lower back, resulting in a deduction of $10 \mathrm{~cm}$ for the mannequin measures. Results showed a slight improvement for all postures and for both scanners when adding the arm-support. When adjusting the virtual height platform to the recommended height, the improvement was substantial and this setup could be considered as acceptable for all positions using the wide bore MRI scanner.

\section{DISCUSSION}

The time-based task analysis revealed that it was possible to reduce the duration of a simulated PTA-IA procedure under MRI guidance when compared the usual duration of fluoroscopy in the conditions described. During the first block of sessions, overall times collected for MRI revealed that the average duration of the procedures using GE iDrive with no communication system installed between the control room and the scanner room, took up to 5 times more than using the RTHawk system with communication. When Bluetooth communication was established, the duration of procedures using GE iDrive still took on average more than twice the length of the fluoroscopy-guided procedure. When using RTHawk, times were comparable to fluoroscopy protocol. However, these times did not take into account several important stages of a usual angioplasty procedure, as indicated in Figure 
1. These stages, mostly regarding the preparation of the patient prior the intervention, would add between 5-10 minutes to the overall duration and are planned to be considered in future investigations. During patient preparation, the equipment available and personnel training are some of the main factors to analyse. In these experiments, we used a dedicated interventional coil prototype "DuoFlex Coil Suite" (GE Healthcare, Waukesha, WI, USA) [14]. Other approaches, such as the use of integrated surface coils for MRI tables should be investigated. Although, the preparation time for MRI might be potentially longer than in fluoroscopy (as it includes the correct placement of the radiofrequency coil), recent studies have shown how acceptable times can be achieved [23]. In this regard, appropriate training of intervention team plays an essential role [24]. When using the iPad as a visualisation device, times were slightly longer in the case of RTiPadBT configuration $(4.25(0.45)$ min for treatment phase) but shorter in the case of GEiPadBT. This can be explained by the lack of familiarity that the clinicians had with this device (i.e., using it for the first time), since RTScreenBT and RTiPadBT configurations were tested before GEScreenBT and GEiPadBT configurations. As reported during the interviews, clinicians detected a small delay (approximately between 1-2 seconds) between the operational handle of devices in the phantom and refresh of the images shown on the iPad screen. This delay was caused by a network problem in the MRI environment setup and was solved. In addition, due to the small sample size collected by clinician, it was not possible to include a statistical evaluation of the difference between clinicians' experience. In future planned experiments, we will include more repetitions with volunteers from different levels of expertise and testing of learning curve using this framework, altering also the order of the configurations to overcome any possible bias that may appear. For instance, since X-Ray procedures were performed in first place, when moving to the MRI setups clinicians might have acquired certain degree of spatial geometry 
familiarisation with the phantom artery, although this was covered with surgical drapes at all times. The future experiments will be able to quantify this.

The CRPA supplemented the performance analysis, providing the capture of all the experimental learning and observation data from the clinician in an unbiased and unobtrusive method. By withholding commentary until the task is completed, it removes risk of contamination of thought and action by concurrent protocols. The offline analysis of video evidence allows for the capture of procedural expertise through viewing of video. By creating a multiplex to view both first and third person perspectives, the capacity of CRPA is maximised. In addition, the results of this study raise the possibility of establishing the optimal form of video demonstration and training elements for new clinical staff. By manipulating the expertise level of clinician, video speed and rapidity of the procedure itself, it can be determined whether the best demonstrator is an expert working normally, or some other form of elaborated or exaggerated demonstration. Recent work in more general tasks such as small object lifting with fingertips - raises an intriguing possibility that it might be more informative to view novice as well as expert behaviour. In a series of experiments, Buckingham and colleagues [25] presented participants with a cube-lifting task and provided training with videos of accurate (expert) behaviour or erroneous lifting behaviour (from novices when weights were uncertain). When they measured the accuracy of the lifting using a biomechanical feedback register, they found better performance for participants who had viewed novice error-prone lifts involving over- and underestimation. This poses the question as to what would constitute the best form of demonstration: error free expert learning or some combination of expert and novice tuition? Perhaps viewing mistakes helps the observer appraise the parameters of the task at hand, in which case these can highlight potential errors that may then be avoided with proactive behaviour. 
By contrast to our study, previous studies that used multi-video recordings in fluoroscopic interventions to evaluate intraprocedural decision-making, focused on third-person perspective, not taking advantage of first-person experience nor paying attention to how limitations in the environment affect performance [27, 28].

The prevalence of low back injuries is a significant concern within the clinical community. Back pain appears as a psychological stressor, leading to medical errors and thereby compromising patient safety. In addition, it has a considerable impact on medical and legal costs $[29,30]$. Therefore, the design of an efficient interventional protocol in a new imaging environment should be accompanied with a study of ergonomic constraints in workplace. Our study takes into consideration one of the most important constraints, which is clinician's posture during procedures, and quantifies it according to the stress caused on the body segments and the muscle work required for such position [20]. Results from the MRI environment indicate that the rooms should be adjusted for its use as interventional facility. The DHM simulation results advise that these adjustments should be customisable depending on the clinician anthropomorphic features (e.g. height, weight, age). Further analyses are likely to follow this work with volunteers from different percentiles of the population. In addition, these studies will apply this framework to evaluate further ergonomic aspects in current angiography suites (e.g. the impact of wearing lead aprons). The authors are aware of the pseudo-subjective ergonomic analysis carried out for these experiments. A new approach, placing sensors in the body during the interventions to record precise parameters of the held postures is being considered. Recent similar approaches in this regards have been done to assess surgeons' positions during laparoscopy procedures [31, 32].

The data collection by hand during the experiments was imprecise and resulted in incomplete datasets leading to the application of the MI method. Although MI is a valid statistical technique, we appreciate the need of mechanisms to prevent these limitations in the future. It 
would be advisable, for instance, to use or develop an electronic application for data gathering using a common and homogeneous terminology. Some of these applications have been shown to be efficient when collecting surgical workflows [32].

In conclusion, a multi-parametric framework is needed in the development of operational protocols for vascular image-guided interventions. A methodology combining a time-based performance evaluation, cognitive assessment of the protocol and ergonomic analysis of the environment, supports the improvement of safety, efficiency and efficacy of image-guided procedures.

Acknowledgments: We thank Lynda Cochrane from the Dundee Epidemiology and Biostatistics Unit (University of Dundee) for her assistance during the statistical analysis, as well as John Ferrut for his support for the ergonomic evaluation. The authors are thankful for financial assistance provided by the FUSIMO ("Patient specific modelling and simulation of focused ultrasound in moving organs") project funded under the European Community's Seventh Framework Programme (FP7/2007-2013) for Research and Technological Development under Grant Agreement no 270186. The Marie Curie Initial Training Network supported this work and the Integrated Interventional Imaging Operating System (IIIOS) project has received funding from the European Community's Seventh Framework Programme (FP7/2007-2013) under Grant Agreement no 238802.

Conflicts of Interest and Source of Funding: The Integrated Interventional Imaging Operating System (IIIOS) project has received funding from the European Community's Seventh Framework Programme (FP7/2007-2013) under Grant Agreement no 238802. Fabiola Fernandez-Gutierrez, Martin A. Rube, Mahsa Fatahi and Benjamin F. Cox have received funding from the European Union (IIIOS project). Helen McLeod, Karen French and Mariana Gueoguieva have received funding from the European Union (FUSIMO project, Grant Agreement no 270186). Kenneth Scott-Brown, Santiago Martinez, Richard D. White 
and Erwin Immel have no conflicts of interest. Graeme J. Houston is director, shareholder, patent holder and receives royalty at Tayside Flow Technologies Ltd. Andreas Melzer is consultant and shareholder at INNOMEDIC GmbH, Herxheim, Germany.

\section{REFERENCES}

[1] M. Saeed and M. Wilson, "Value of MR contrast media in image-guided body interventions.," World journal of radiology, vol. 4, no. 1, pp. 1-12, Jan. 2012.

[2] M. Bock and F. K. Wacker, "MR-guided intravascular interventions: techniques and applications.," Journal of magnetic resonance imaging : JMRI, vol. 27, no. 2, pp. 32638, Feb. 2008.

[3] S. Kos, R. Huegli, G. M. Bongartz, A. L. Jacob, and D. Bilecen, "MR-guided endovascular interventions: a comprehensive review on techniques and applications.," European radiology, vol. 18, no. 4, pp. 645-57, Apr. 2008.

[4] A. Losey, P. Lillaney, A. Martin, and D. Cooke, "Magnetically Assisted Remotecontrolled Endovascular Catheter for Interventional MR Imaging: In Vitro Navigation at 1.5 T versus X-ray Fluoroscopy,” Radiology, vol. 271, no. 3, pp. 862-869, 2014.

[5] J.-C. Gentric, B. Trelhu, P. Jannin, L. Riffaud, J.-C. Ferré, and J.-Y. Gauvrit, "Development of workflow task analysis during cerebral diagnostic angiographies: Time-based comparison of junior and senior tasks.," Journal of neuroradiology. Journal de neuroradiologie, pp. 1-6, Jul. 2013.

[6] R. Flin, S. Yule, L. McKenzie, S. Paterson-Brown, and N. Maran, "Attitudes to teamwork and safety in the operating theatre.," The surgeon: journal of the Royal Colleges of Surgeons of Edinburgh and Ireland, vol. 4, no. 3, pp. 145-51, Jun. 2006.

[7] S. Johnson, a Healey, J. Evans, M. Murphy, M. Crawshaw, and D. Gould, "Physical and cognitive task analysis in interventional radiology.," Clinical radiology, vol. 61, no. 1, pp. 97-103, Jan. 2006.

[8] I. Van Herzeele, R. Aggarwal, S. Neequaye, A. Darzi, F. Vermassen, and N. J. Cheshire, "Cognitive training improves clinically relevant outcomes during simulated endovascular procedures.," Journal of vascular surgery, vol. 48, no. 5, pp. 1223-30, Nov. 2008.

[9] T. van Gog, F. Paas, J. J. G. van Merriënboer, and P. Witte, "Uncovering the problemsolving process: cued retrospective reporting versus concurrent and retrospective reporting.," Journal of experimental psychology. Applied, vol. 11, no. 4, pp. 237-44, Dec. 2005.

[10] J. H. Brusin, "Ergonomics in Radiology," Journal of the American Society of Radiologic Technologists, vol. 83, no. 2, pp. 141-157, 2011. 
[11] a. García-Lallana, G. Viteri-Ramírez, R. Saiz-Mendiguren, J. Broncano, and J. Dámaso Aquerreta, "Ergonomics of the workplace in radiology," Radiología (English Edition), vol. 53, no. 6, pp. 507-515, Nov. 2011.

[12] C. J. J. M. Sikkink, M. M. P. J. Reijnen, and C. J. Zeebregts, "The creation of the optimal dedicated endovascular suite.," European journal of vascular and endovascular surgery: the official journal of the European Society for Vascular Surgery, vol. 35, no. 2, pp. 198-204, Feb. 2008.

[13] B. Rostenberg and P. R. Barach, "Design of cardiovascular operating rooms for tomorrow's technology and clinical practice — Part one," Progress in Pediatric Cardiology, vol. 33, no. 1, pp. 57-65, Dec. 2011.

[14] M. A. Rube, F. Fernandez-Gutierrez, B. F. Cox, A. B. Holbrook, J. G. Houston, R. D. White, H. McLeod, M. Fatahi, and A. Melzer, "Preclinical feasibility of a technology framework for MRI-guided iliac angioplasty.," International journal of computer assisted radiology and surgery, no. [Epub ahead of print], Aug. 2014.

[15] D. Chaffin, "Digital Human Modeling for Workspace Design," Reviews of Human Factors and Ergonomics, vol. 4, no. 1, pp. 41-74, 2008.

[16] J. M. Santos, G. a Wright, and J. M. Pauly, "Flexible real-time magnetic resonance imaging framework.," Conference proceedings : ... Annual International Conference of the IEEE Engineering in Medicine and Biology Society. IEEE Engineering in Medicine and Biology Society. Conference, vol. 2, pp. 1048-51, Jan. 2004.

[17] G. a. Ballinger, "Using Generalized Estimating Equations for Longitudinal Data Analysis," Organizational Research Methods, vol. 7, no. 2, pp. 127-150, Apr. 2004.

[18] A. M. Law, Simulation Modeling and Analysis, Forth Edit. Mcgraw Hill Higher Education, 2007.

[19] Y. He, "Missing data analysis using multiple imputation: getting to the heart of the matter.," Circulation. Cardiovascular quality and outcomes, vol. 3, no. 1, pp. 98-105, Jan. 2010.

[20] L. McAtamney and E. Nigel Corlett, "RULA: a survey method for the investigation of work-related upper limb disorders.," Applied ergonomics, vol. 24, no. 2, pp. 91-9, Apr. 1993.

[21] W. E. Woodson, B. Tillman, and P. Tillman, Human Factors Design Handbook, 2nd Editio. New York: McGraw Hill, Inc, 1992.

[22] F. Fernandez-Gutierrez, J. Ferut, J. Smink, G. Houston, and A. Melzer, "Ergonomics for MRI guided procedures. Case of study: postural analysis for MRI scanners," Proceedings of the 27th International Congress and Exhibition. International Journal of Computer Assisted Radiology and Surgery, vol. 8, no. S1, pp. 5-11, May 2013. 
[23] T. Takahara, T. Kwee, S. Kibune, R. Ochiai, T. Sakamoto, T. Niwa, M. Van Cauteren, and P. Luijten, "Whole-body MRI using a sliding table and repositioning surface coil approach.," European radiology, vol. 20, no. 6, pp. 1366-73, Jun. 2010.

[24] J. Kettenbach, D. F. Kacher, A. R. Kanan, B. Rostenberg, J. Fairhurst, A. Stadler, K. Kienreich, and F. a Jolesz, "Intraoperative and interventional MRI: recommendations for a safe environment.," Minimally invasive therapy \& allied technologies : MITAT : official journal of the Society for Minimally Invasive Therapy, vol. 15, no. 2, pp. 5364, Jan. 2006.

[25] G. Buckingham, J. D. Wong, M. Tang, P. L. Gribble, and M. a Goodale, "Observing object lifting errors modulates cortico-spinal excitability and improves object lifting performance.," Cortex; a journal devoted to the study of the nervous system and behavior, pp. 1-10, Jul. 2013.

[26] J. R. Duncan, B. Kline, and C. B. Glaiberman, "Analysis of simulated angiographic procedures. Part 2: extracting efficiency data from audio and video recordings.," Journal of vascular and interventional radiology: JVIR, vol. 18, no. 4, pp. 535-44, Apr. 2007.

[27] E. Beta, A. S. Parikh, M. Street, and J. R. Duncan, "Capture and analysis of data from image-guided procedures.," Journal of vascular and interventional radiology : JVIR, vol. 20, no. 6, pp. 769-81, Jun. 2009.

[28] L. W. Klein, D. L. Miller, S. Balter, W. Laskey, D. Haines, A. Norbash, M. A. Mauro, and J. A. Goldstein, "Occupational Health Hazards in Time for a Safer Environment," vol. 250, no. 2, pp. 538-544, 2009.

[29] M. a Mohseni-Bandpei, M. Ahmad-Shirvani, N. Golbabaei, H. Behtash, Z. Shahinfar, and C. Fernández-de-las-Peñas, "Prevalence and risk factors associated with low back pain in Iranian surgeons.," Journal of manipulative and physiological therapeutics, vol. 34, no. 6, pp. 362-70, 2011.

[30] F. J. Pérez-Duarte, M. Lucas-Hernández, a Matos-Azevedo, J. a Sánchez-Margallo, I. Díaz-Güemes, and F. M. Sánchez-Margallo, "Objective analysis of surgeons' ergonomy during laparoendoscopic single-site surgery through the use of surface electromyography and a motion capture data glove.," Surgical endoscopy, vol. 28, no. 4, pp. 1314-20, Apr. 2014.

[31] K. H. Kramp, M. J. van Det, E. R. Totte, C. Hoff, and J.-P. E. N. Pierie, "Ergonomic assessment of the French and American position for laparoscopic cholecystectomy in the MIS Suite.," Surgical endoscopy, vol. 28, no. 5, pp. 1571-8, May 2014.

[32] T. Neumuth, "Neumuth Validation of KA for Surgical Process Models JAMIA 2009 Validation of KA for Surgical Process Models JAMIA 2009 Page 2 of 27," vol. 16, no. 1, pp. 72-80, 2009.

\section{APPENDIX}




\section{Facilities and equipment:}

Fluuroscopy-guided interventions were performed on a digital subtraction angiography (DSA) unit (OEC 9900 Elite, GE Medical Systems, Waukesha, WI, USA). MRI-guided interventions used a 1.5T MRI scanner (Signa HDxt, GE Medical Systems, Waukesha, WI, USA).

The experimental setups were all conducted on an arterial vessel phantom consisting of linked femoral, abdominal and thoracic module (L-F-S-Left-003, A-S-N-001, T-R-N-020, Elastrat, Sarl, Switzerland). The phantom was connected to a heart-lung machine (HL-30, Maquet, Rastatt, Germany), customising one HL-30 D150 pump to mimic (pulsatile) physiologic flow. Plastic tubes were taken from the phantom to the heart-lung machine, which was in an annex room. Tubes passed the Faraday cage through the wave- guides. Silicon tubing (PT 12.7x3.2, Silex, Bordon, UK), with an inner diameter of $16 \mathrm{~mm}$ and length of $5 \mathrm{~m}$ was used. An arterial blood pressure monitoring kit with a trace was also used to examine systolic/diastolic pressures during the interventions. A permanent introducer sheath (12F) was inserted into the femoral artery to provide access and exchange of devices during the interventions. A neonatal blood pressure cuff (SoftCheck Neonatals, Statcorp Medical, Jacksonville, FL, USA) was secured to the right common iliac artery (with electrical tape and rubber sheet) to mimic stenosis (See Appendix-Figure).

The two workstations used in the MRI, standard control console (software release 15.0M4A, GE Healthcare, Waukeska, WI, USA) and the real-time MRI software framework (RTHawk, Version 0.9.28, HeartVista, Inc., Los Altos, CA, USA) were in communication via Gigabit Ethernet and were connected via optical fibre cables (M1-1000, Opticis, Sungnam City, Korea) to a shielded 40" LCD monitor (Multeos 401, NEC Corporation, Tokyo, Japan) to display the MR images inside the MRI scanner room.

IP cameras models were: M1011w and M1031w, Axis Communications, Lund, Sweden. 
Recoding spectacles were PivotHead, models Durango Chameleon and Recon Black Jet frames with no lenses fitted (Cape Evolution Ltd, Greenwood Village, CO, USA).

Second tablet device used for scanner/control room communication was an iPad 3 (Apple Inc., Cupertino, CA, US) and the Bluetooth earphones were Calisto B70 (Plantronics, Santa Cruz, CA, USA).

Devices:

We customised commercially available non-braided balloon catheters (5F PTA Balloon catheter, Workhorse II, AngioDynamics, Lathan, NY, US) by attaching a resonant circuit $5 \mathrm{~mm}$ distally to the inflatable balloon. Each resonant circuit was tuned to $63.8 \mathrm{MHz}$ (the proton Larmor frequency at $1.5 \mathrm{~T}$ ) in $0.9 \%$ saline solution.

Additional devices used during the interventions included:

- 5-F Straight catheter (BeaconTipRoyal Flush,CookInc., Bloomington, IN, USA), length 70cm (lumen 0.035”)

- 6-F Multipurpose catheter (Soft-Vu, AngioDynamics, Latham, NY, USA), length 90cm (lumen 0.035”)

- For fluoroscopy, commercially available 0.035" guidewires (Standard Glidewire, Terumo, Somer- set, NJ, USA) were used.

- For MRI, a novel hydrophilic-coated and MRI-safe guidewire prototype that was developed with EPflex GmbH (Dettingen/Erms, Germany) was used, with a diameter of $0.035 "$ and a length of $120 \mathrm{~cm}$.

A detailed description of the fabrication of this devices can be read in Rube et al [14].

Figure: 
a) Fluoroscopy-guided protocol
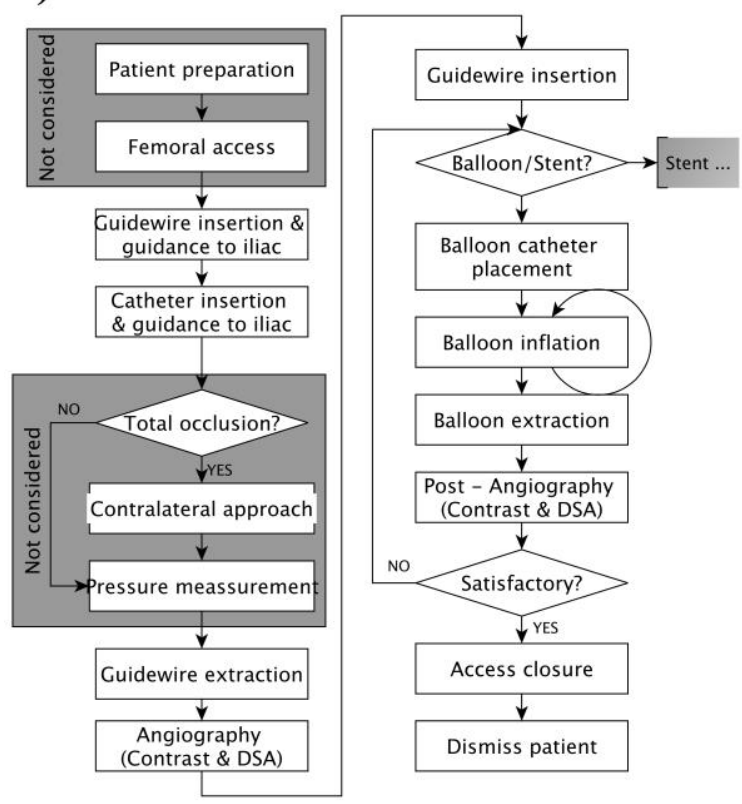

DSA - Digital Subtraction Angiography b) MRI-guided protocol

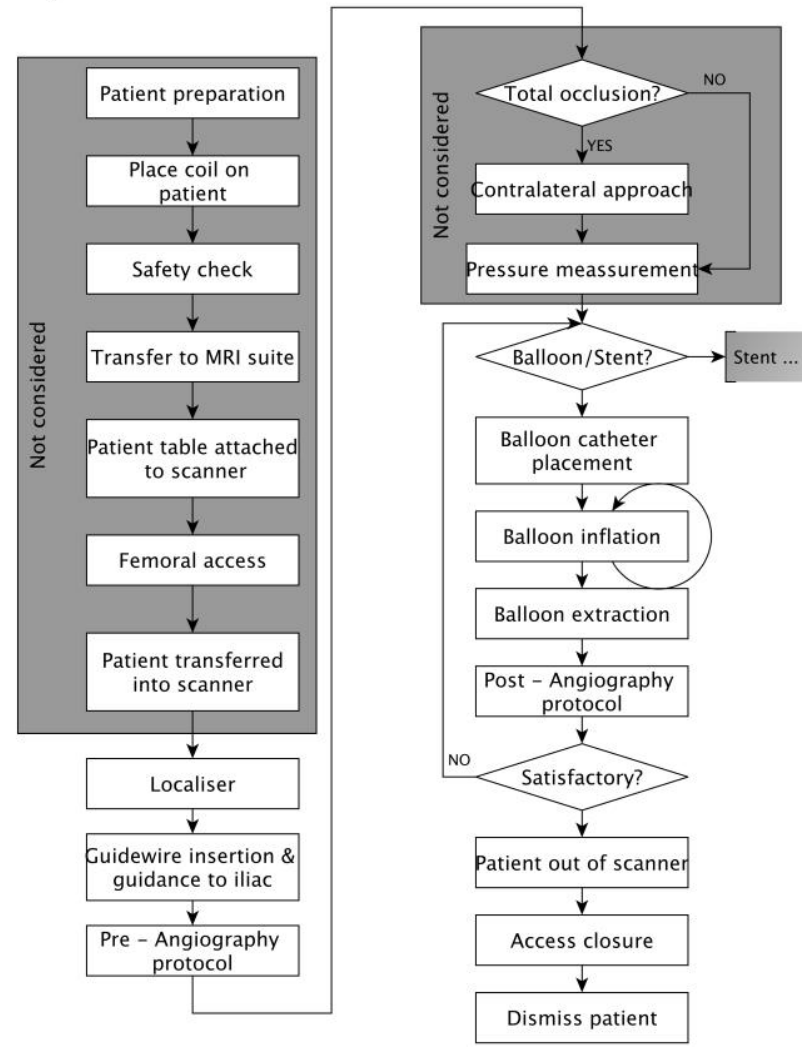

FIGURE 1. Percutaneous transluminal angioplasties of the iliac artery (PTA-IA) conceptual workflows for the iliac artery under fluoroscopy (a) and under MRI guidance (b) followed during the experiments. The grey areas indicate the tasks that were not considered for the study as not relevant for the interventional tasks. 

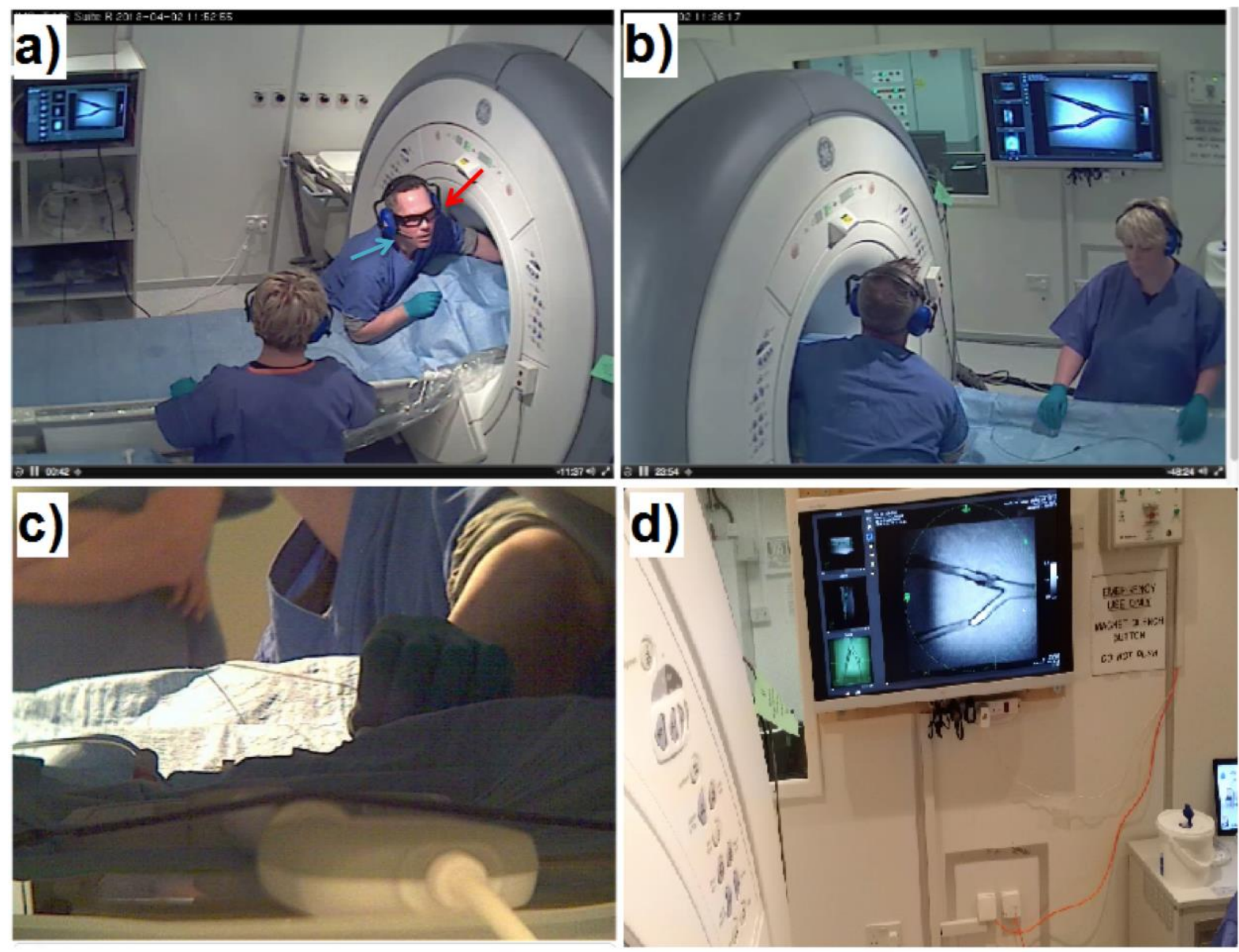

FIGURE 2. Perspectives of the cameras arranged in the MRI suite during the interventions: (a) right, (b) left, (c) bore, (d) first-person. Red arrow indicates the recording spectacles (PivotHead, models Durango Chameleon and Recon Black Jet frames with no lenses fitted, Cape Evolution Ltd, Greenwood Village, CO, USA) used for first-person experience evaluation. Blue arrow indicates the Bluetooth earphones (Calisto B70, Plantronics, Santa Cruz, CA, USA) under the noise protection earmuffs. 


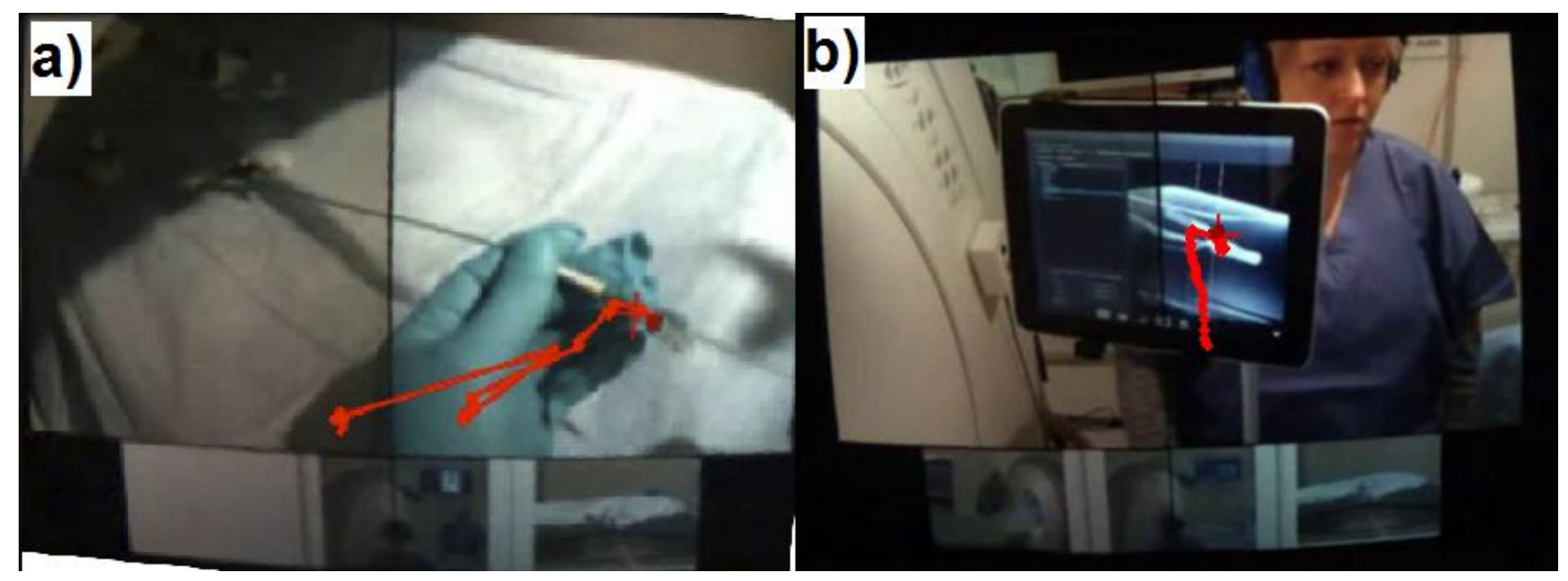

FIGURE 3. CRPA interviews with clinicians. Figures (a) and (b) illustrate the first and second person perspectives (lower part of the images) with overlaid gaze cursor. This red cursor shows the location of the eye gaze on the image for the current location and the previous .25 of a second.

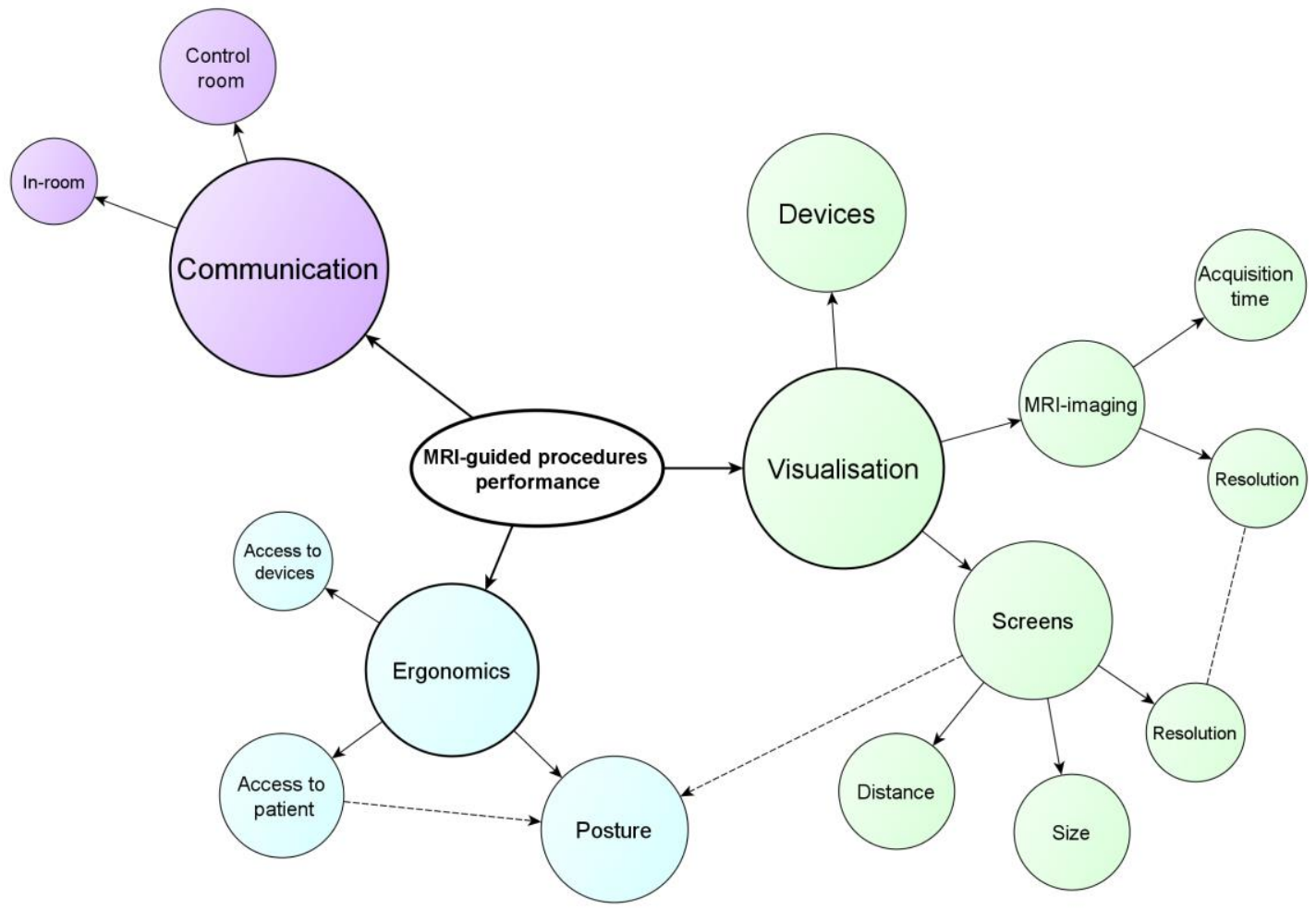

FIGURE 4. CRPA diagram illustrating main factors that affect an intervention according to the clinicians' feedback. The size of a bubble represents the importance level given by the clinicians during the interviews: A larger bubble means higher importance. Black arrows 
represent the hierarchy within a group. A red arrow indicates an interrelation between factors of two different groups.

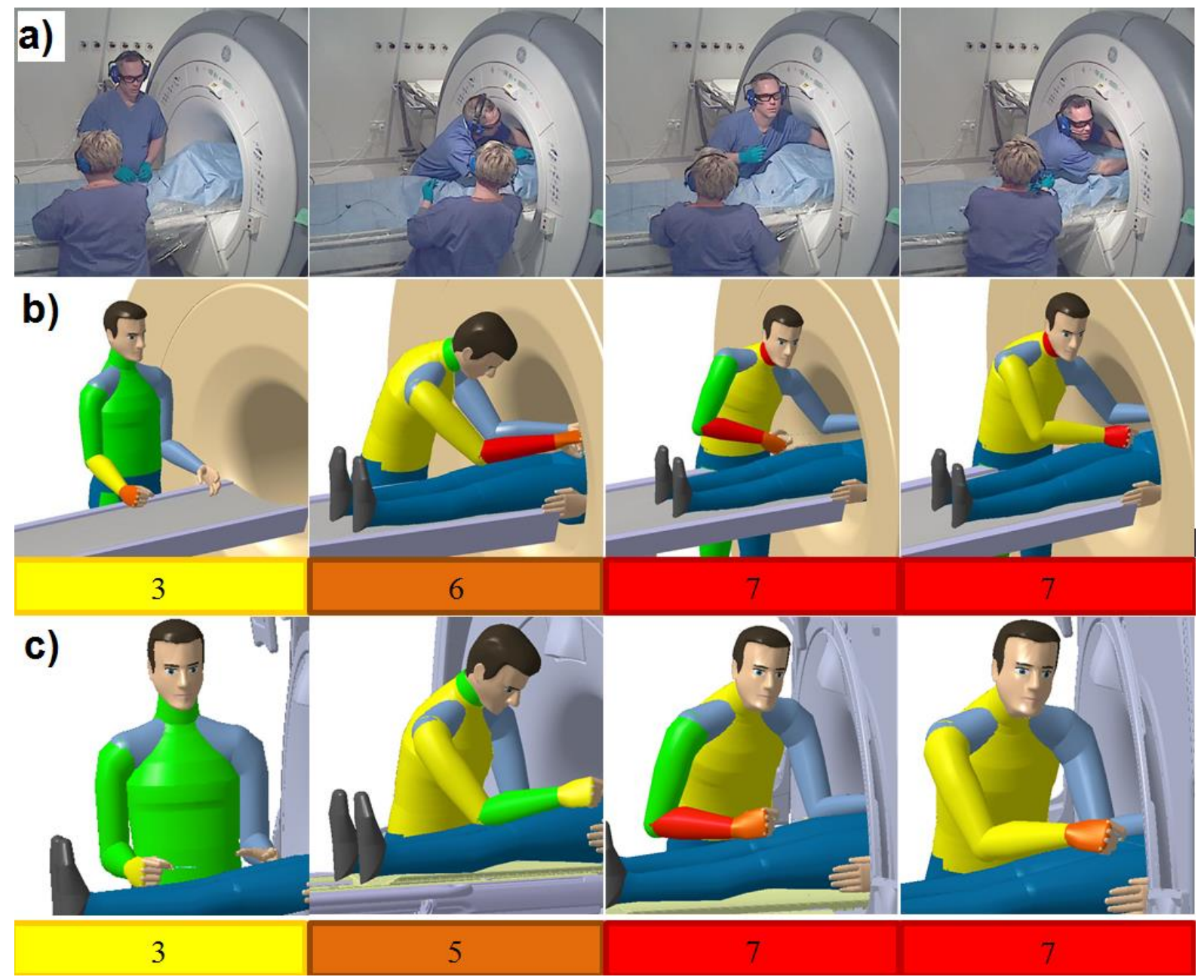

FIGURE 5. Key positions (postures) defined for MRI-guided procedures (a, first row, from left to right - positions 1 to 4), equivalent postures modelled in Delmia V5R20 for the 1.5T GE Signa MRI scanner (b, second row) and the 3T GE Discovery MRI scanner (c, third row) (GE Healthcare, Waukesha, WI, USA). Below both virtual environments, the global scores given by the RULA analysis are shown. 


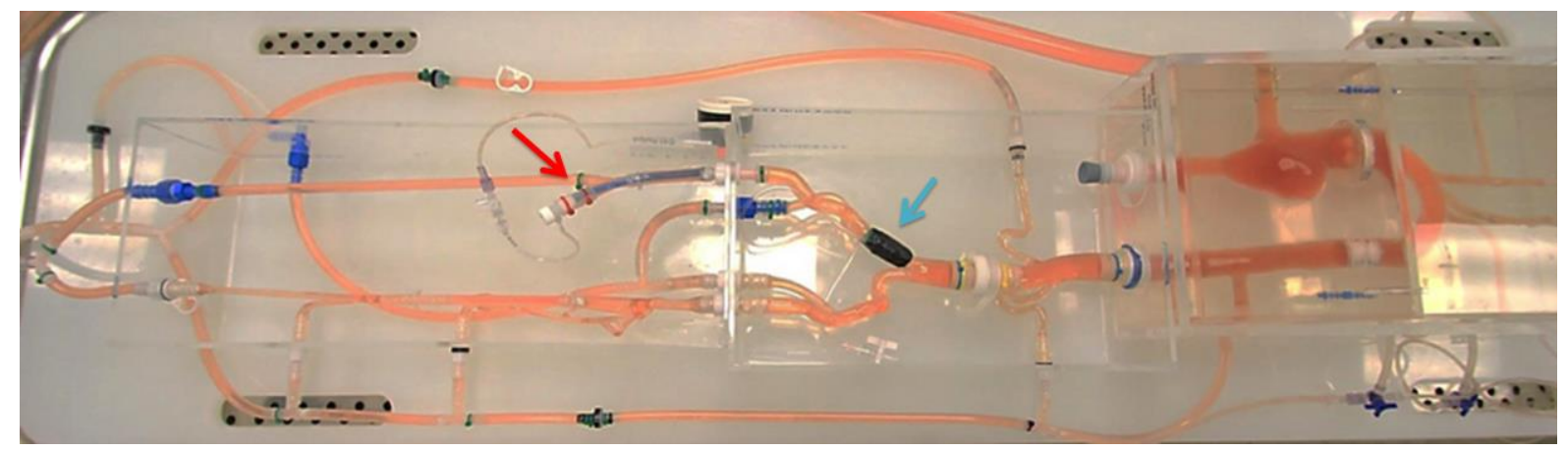

APPENDIX-FIGURE. Fully perfused thorax to above the knee vascular phantom (Elastrat, Sarl, Switzerland). Red arrow indicates the 12F sheath introducer used for permanent access. Blue arrow indicates a neonatal pressure cuff (SoftCheck Neonatals, Statcorp Medical, Jacksonville, FL, USA) that was attached to the right common iliac artery to mimic a stenosis

Table:

\begin{tabular}{|l|l|l|l|}
\hline Configuration & $\begin{array}{l}\text { Communication with } \\
\text { Control Room }\end{array}$ & Workstation & Visualisation \\
\cline { 1 - 1 } I - GEScreenBT & Bluetooth & Standard & In-room monitor \\
\cline { 1 - 1 } II - GEiPadBT & & & iPad \\
\cline { 1 - 1 } III - RTScreenBT & & RTHawk & In-room monitor \\
\cline { 4 - 4 } IV - RTiPadBT & & & iPad \\
\hline V - GEScreen & None & Standard & In-room monitor \\
\hline VI - RTScreen & None & RTHawk & In-room monitor \\
\hline
\end{tabular}

TABLE 1. MRI configurations evaluated. First column shows number and acronyms given to each configuration.

\begin{tabular}{|c|c|c|c|}
\hline \multirow{2}{*}{ Configuration } & \multicolumn{2}{|c|}{ Total duration per clinician (Mean(SE)) (min) } & $\begin{array}{c}\text { Overall Total } \\
\text { Duration (Mean } \\
\text { (SE)) (min) }\end{array}$ \\
\cline { 2 - 4 } & Clinician A & Clinician B & $8.49(0.75)$ \\
\hline Fluoroscopy (baseline) & $7.47(0.77)$ & $9.53(1.08)$ & $18.09(0.57)$ \\
\hline GEScreenBT & $17.82(0.96)$ & $18.36(0.94)$ & $17.19(0.73)$ \\
\hline GEiPadBT & $16.37(0.14)$ & $18.43(1.66)$ & $8.56(0.64)$ \\
\hline RTScreenBT & $7.32(0.07)$ & $9.39(0.72)$ & $9.48(1.13)$ \\
\hline RTiPadBT & $7.71(1.17)$ & $11.25(0.13)$ & \\
\hline
\end{tabular}

TABLE 2. Total procedure times in minutes for fluoroscopy guided procedures and MRI configurations evaluated (see acronyms description in Table 1) during the second block of sessions, per clinician and overall. All the times are expressed in mean (standard error). 


\begin{tabular}{|c|c|c|c|}
\hline Configuration & $\begin{array}{c}\text { Treatment } \\
\text { (Mean (SE)) (min) }\end{array}$ & $\begin{array}{c}\text { Pre-angiography } \\
\text { (Mean (SE) }(\mathbf{m i n})\end{array}$ & $\begin{array}{c}\text { Post-angiography } \\
\text { (Mean (SE)) (min) }\end{array}$ \\
\hline Fluoroscopy (baseline) & $3.63(0.27)$ & $1.71(0.24)$ & $1.94(0.25)$ \\
\hline GEScreenBT & $3.23(0.25)$ & $6.28(0.05)$ & $5.61(0.11)$ \\
\hline GEiPadBT & $3.14(0.28)$ & $6.00(0.14)$ & $5.70(0.31)$ \\
\hline RTScreenBT & $3.33(0.35)$ & $1.34(0.18)$ & $1.72(0.17)$ \\
\hline RTiPadBT & $4.25(0.45)$ & $1.38(0.01)$ & $1.47(0.04)$ \\
\hline
\end{tabular}

TABLE 3. Average durations per configuration for the phases of treatment, pre-angiography and post-angiography protocols. See configuration acronyms in Table 1.

\begin{tabular}{|c|c|c|c|c|c|c|c|c|}
\hline & \multicolumn{4}{|c|}{ 1.5T GE Signa (60cm bore) } & \multicolumn{4}{|c|}{ 3T GE Discovery (70 cm bore) } \\
\hline & Initial & $\begin{array}{l}\text { Arm- } \\
\text { Support }\end{array}$ & $\begin{array}{l}\text { Height } \\
\text { platform }\end{array}$ & $\begin{array}{l}\text { Combined } \\
\text { effect }\end{array}$ & Initial & $\begin{array}{l}\text { Arm- } \\
\text { Support }\end{array}$ & $\begin{array}{l}\text { Height } \\
\text { platform }\end{array}$ & $\begin{array}{l}\text { Combined } \\
\text { effect }\end{array}$ \\
\hline Position 1 & 3 & 3 & 3 & 3 & 3 & 3 & 3 & 3 \\
\hline Position 2 & 6 & 6 & 5 & 4 & 5 & 4 & 3 & 3 \\
\hline Position 3 & 7 & 6 & 3 & 3 & 7 & 6 & 3 & 3 \\
\hline Position 4 & 7 & 6 & 6 & 5 & 7 & 6 & 3 & 3 \\
\hline
\end{tabular}

adjustable height platform and a combination of the two factors for both scanners. 